$<$ Original Article $>$

\title{
Anti-diabetic effects of benfotiamine on an animal model of type 2 diabetes mellitus
}

\author{
Kang Min Chung, Wonyoung Kang, Dong Geon Kim, Hyun Ju Hong, Youngjae Lee, Chang-Hoon Han* \\ Department of Toxicology and Biochemistry, College of Veterinary Medicine, Jeju National University, Jeju 690-756, Korea
}

(Received: November 2, 2013; Accepted: January 24, 2014)

\begin{abstract}
Although benfotiamine has various beneficial anti-diabetic effects, the detailed mechanisms underlying the impact of this compound on the insulin signaling pathway are still unclear. In the present study, we evaluated the effects of benfotiamine on the hepatic insulin signaling pathway in Otsuka Long-Evans Tokushima Fatty (OLETF) rats, which are a type 2 diabetes mellitus model. OLETF rats treated with benfotiamine showed decreased body weight gain and reduced adipose tissue weight. In addition, blood glucose levels were lower in OLETF rats treated with benfotiamine. Following treatment with benfotiamine, the levels of Akt phosphorylation (S473/T308) in the OLETF groups increased significantly compared to the OLETF control group so that they were almost identical to the levels observed in the control group. Moreover, benfotiamine restored the phosphorylation levels of both glycogen synthase kinase (GSK)-3 $\alpha / \beta$ (S21, S9) and glycogen synthase (GS; S641) in OLETF rats to nearly the same levels observed in the control group. Overall, these results suggest that benfotiamine can potentially attenuate type 2 diabetes mellitus in OLETF rats by restoring insulin sensitivity through upregulation of Akt phosphorylation and activation of two downstream signaling molecules, GSK- $3 \alpha / \beta$ and GS, thereby reducing blood glucose levels through glycogen synthesis.
\end{abstract}

Keywords : Akt phosphorylation, benfotiamine, insulin signaling pathway, OLETF rats, type 2 diabetes mellitus

\section{Introduction}

The prevalence of diabetes mellitus and complications associated with this disease are rapidly increasing throughout the world because of changes in lifestyle and increased life spans [13]. Diabetes mellitus is a metabolic disease characterized by hyperglycemia that is linked to various disorders such as neuropathy, nephroopathy, and retinopathy [7]. These complications are important causes of increasing death rates among diabetes mellitus patients [9]. Type 1 diabetes results from a failure of the pancreas to produce insulin whereas type 2 diabetes is caused by insulin resistance. Type 2 diabetic patients develop metabolic syndrome more frequently than individuals with type 1 diabetes, and patients with metabolic syndrome have a high mortality rate [28].

Insulin resistance is a physiological condition characterized by an impaired ability of insulin to regulate blood glucose levels [1] and has been linked to oxidative stress [20, 21]. An excess of glucose products energizing mitochondria results in oxidative stress due to the accumulation of reactive oxygen species (ROS) [8, 11, 18, 19]. One type of ROS, superoxides, switches off the insulin response [8, 18]. Insulin resistance can thus be viewed as a defense mechanism against cellular oxidation damage by blocking increased glucose transport into cells in response to insulin $[14,26]$. In the liver, however, insulin resistance causes inactivation of glycogenesis, the process of synthesizing glycogen from glucose [27]. Under these conditions, glucose cannot be converted into glycogen, blood glucose levels gradually increase, and insulin resistance is elevated [27].

Benfotiamine, a lipid soluble thiamine analogue, has greater bioavailability than thiamine and is commonly used to treat diabetic vascular complications [2, 12, 25]. This compound is a thiamine-dependent transketolase activator that directs glucose substrate to the pentose phosphate pathway. Thus, it blocks three major pathways associated with hyperglycemic damage: the hexosamine, advanced glycation end product (AGE) formation, and diacylglycerol (DAG)-protein kinase $\mathrm{C}(\mathrm{PKC})$ pathways $[12,25]$. All of these mechanisms consequently induce superoxide overproduction by the mitochondrial electron transport chain that causes vascular dysfunction $[12,22,23]$. Although benfotiamine blocks the pathways producing superoxides, no study has demonstrated the effect of benfotiamine on insulin resistance in an animal model of type 2 diabetes mellitus. The Otsuka Long Evas Tokushima Fatty (OLETF) rat is a genetic model of type 2 diabetes mel-

*Corresponding author

Tel: +82-64-754-3378, Fax: +82-64-756-3354

E-mail: chhan@jejunu.ac.kr 
litus with obesity. OLETF rats show clinically relevant type 2 diabetic phenotypes such as insulin resistance, hyperinsulinemia, hyperglycemia, and mild obesity [15]. In the present study, we evaluated the anti-diabetic effects of benfotiamine on the hepatic insulin signaling pathway in OLETF rats, a well-known genetic model of type 2 diabetes mellitus.

\section{Materials and Methods}

\section{Materials}

Benfotiamine was purchased from Dalim BioTech (Korea). Anti-phospho-Akt (S473; polyclonal), anti-phospho-Akt (T308; polyclonal), anti-phospho-GSK-3 $\alpha / \beta$ (S21/9; polyclonal), antiGSK-3 $\alpha / \beta$ (monoclonal), anti-phospho-GS (S641; polyclonal), and anti-GS (polyclonal) antibodies were purchased from Cell Signaling Technology (USA). Anti- $\beta$-actin (monoclonal) antibody was from Sigma (USA). Anti-Akt (polyclonal), anti-rabbit immunoglobulin G (IgG), and anti-mouse immunoglobulin G (IgG) horseradish peroxidase (HRP)-linked secondary antibodies were from Santa Cruz Biotechnology (USA).

\section{Animal experiments}

Male OLETF and LETO rats were purchased from Otsuka Pharmaceutical (Japan). The animals were housed in rooms kept at $24 \pm 1{ }^{\circ} \mathrm{C}$ with $50 \%$ humidity and a 12-h light/dark cycle. The rats were fed a normal diet (5L79; Orient, Korea). The OLETF rats, 27 week old and weighing 540 740 g, were divided into three groups: untreated $(n=7)$, treatment with $100 \mathrm{mg} / \mathrm{kg}$ benfotiamine $(\mathrm{n}=7)$, and treatment with $200 \mathrm{mg} /$ $\mathrm{kg}$ benfotiamine $(\mathrm{n}=7)$. LETO rats, 27 week old and weighing $420 \sim 520 \mathrm{~g}$, served as controls $(\mathrm{n}=7)$. Benfotiamine was administered with tap water to the OLETF rats for 7 week. Body weight, food consumption and water intake were recorded once a week. All animal experiments were carried out in accordance with the National Institute of Health "Guide for the Care and Use of Laboratory Animals", and were approved by the Institutional Animal Care and Use Committee of Jeju National University (ACUCC; Approval No. 20100016).

\section{Measurement of blood glucose levels}

Prior to the experiment (time 0), blood samples were collected from the tail vein after the animals were fasted for 16 h. Blood glucose levels were measured using a OneTouch Horizon system (LifeScan, USA). After treatment with benfotiamine for 7 week, blood glucose levels were again measured using the same methods.

\section{Tissue and organ isolation}

At the end of study ( 7 week), the rats were fasted for $16 \mathrm{~h}$ and euthanized in $\mathrm{CO}_{2}$ gas chamber. The heart, descending aorta, liver, kidneys, epididymal adipose tissue, and gastrocnemius muscle were removed, weighed, minced, frozen in liquid nitrogen, and stored at $-80^{\circ} \mathrm{C}$ until use.

\section{Sample preparation}

Approximately $100 \mathrm{mg}$ of frozen liver were placed in 1.2 $\mathrm{mL}$ lysis buffer [20 mM Tris-HCl, (pH 7.4) with $5 \mathrm{mM}$ EDTA (pH 8.0), $10 \mathrm{mM} \mathrm{Na}_{2} \mathrm{P}_{4} \mathrm{O}_{7}, 100 \mathrm{mM} \mathrm{NaF}, 2 \mathrm{mM} \mathrm{Na}_{3} \mathrm{VO}_{4}, 1 \%$ NP-40, $13.2 \mu \mathrm{g} / \mathrm{mL}$ leupeptin, and $1 \mathrm{mM} \mathrm{PMSF}]$ and homogenized for $90 \mathrm{sec}$ at $11,000 \mathrm{rpm}$ with a tissue homogenizer. The homogenates were centrifuged at $800 \times \mathrm{g}$ for $15 \mathrm{~min}$. Supernatants were removed and centrifuged at $10,000 \times \mathrm{g}$ for $20 \mathrm{~min}$ at $4^{\circ} \mathrm{C}$. The supernatants from the second centrifugation were stored at $-80^{\circ} \mathrm{C}$ until use. Protein concentrations were measured using the Bradford method [3].

\section{Western blot analysis}

Aliquots of liver homogenate protein $(60 \mu \mathrm{g})$ were separated in a $10 \%(\mathrm{v} / \mathrm{v})$ SDS-PAGE gel and transferred onto PVDF membranes (Immobilon P; Millipore, USA) for 100 $\min$ at $110 \mathrm{~V}$. The membrane was incubated at room temperature for $1 \mathrm{~h}$ in blocking buffer $[5 \%(\mathrm{w} / \mathrm{v})$ non-fat dry milk in TTBS; $20 \mathrm{mM}$ Tris-HCl (pH 7.6) with $137 \mathrm{mM} \mathrm{NaCl}$ and $0.05 \%$ Tween-20], washed four times in TTBS (10 min per wash), and incubated for $1 \mathrm{~h}$ with the following primary antibodies diluted $1: 1,000$ in TTBS : anti-p-Akt (S473), anti-pAkt (T308), anti-Akt, anti-p-GSK-3 $\alpha / \beta$ (S21/S9), anti-GSK$3 \alpha / \beta$, anti-p-GS (S641), anti-GS, and anti- $\beta$-actin. After washing with TTBS, the membranes were incubated for 40 min with anti-rabbit IgG or anti-mouse IgG HRP-linked secondary antibodies diluted $1: 10,000$ or $1: 5,000$ in TTBS. Reactive bands were visualized using enhanced chemiluminesence (ECL) reagents (Amersham Bioscience, USA). The band intensities were analyzed with Image J 1.42 software (National Institute of Mental Health, USA).

\section{Statistical analysis}

All results were represented as the mean \pm SEM. Data were analyzed by one way analysis of variance (ANOVA) using SPSS program (SPSS, USA). When the ANOVA test yielded statistical differences $(p<0.05$ or 0.01$)$, Dunnett's two-tailed $t$-test was used to compare each group against the control group. The value of $p<0.05$ was used as the criterion for statistical significance.

\section{Results}

\section{Body weight gain}

The effect of benfotiamine on body weight was evaluated once a week. Weight gain of the OLETF control rats was significantly higher $(p<0.01)$ than that of the LETO animals at the end of the study ( 7 weeks; Table 1 ). In contrast, increases in body weight of the OLETF rats treated with both concentrations of benfotiamine $(100 \mathrm{mg} / \mathrm{kg}$ and $200 \mathrm{mg} / \mathrm{kg})$ were significantly lower ( $p<0.05$ and $p<0.01$, respectively) than those of the OLETF control group. In particular, administration of benfotiamine $(100 \mathrm{mg} / \mathrm{kg}$ or $200 \mathrm{mg} / \mathrm{kg})$ significantly reduced adipose tissue weight $(p<0.05$; Table 1$)$. These results indicate that benfotiamine decreased body weight gain 
Table 1. Effect of benfotiamine on the weight of body, adipose tissue, and muscle

\begin{tabular}{lcccc}
\hline \hline \multicolumn{1}{c}{ Measurement } & LETO & OLETF & OLETF B100 & OLETF B200* $^{*}$ \\
\hline Final body weight $(\mathrm{g})^{\S}$ & $464 \pm 17.2$ & $658 \pm 22.3^{*}$ & $608 \pm 25.7^{\#}$ & $605 \pm 19.4^{\#}$ \\
Body weight gain $(\mathrm{g})$ & $49.6 \pm 5.2$ & $97.5 \pm 12.2^{*}$ & $67.0 \pm 7.1^{\#}$ & $57.2 \pm 10.2^{\# \#}$ \\
Adipose tissue $(\mathrm{g})^{\S}$ & $7.0 \pm 0.4$ & $44.8 \pm 4.6^{*}$ & $32.9 \pm 4.7^{\#}$ & $34.8 \pm 3.3^{\#}$ \\
Gastrocnem. muscle $(\mathrm{g})^{\S}$ & $12.3 \pm 0.6$ & $16.4 \pm 0.7$ & $15.5 \pm 0.5$ & $14.2 \pm 1.3$ \\
Food intake (g/week) & $27.7 \pm 0.3$ & $39.8 \pm 1.8^{*}$ & $37.4 \pm 1.3^{*}$ & $36.2 \pm 1.5^{*}$ \\
Water intake (mL/week) & $35.8 \pm 1.8$ & $39.3 \pm 2.1$ & $37.3 \pm 2.0$ & $38.7 \pm 2.3$ \\
\hline
\end{tabular}

Data are presented as the mean \pm SE. ${ }^{*} p<0.01 v s$. the LETO rats; ${ }^{\#} p<0.05$ and ${ }^{\# \#} p<0.01 v s$. the OLETF control. OLETF treated with $100 \mathrm{mg} / \mathrm{kg}$ benfotiamine. "OLETF treated with $200 \mathrm{mg} / \mathrm{kg}$ benfotiamine. ${ }^{\S}$ Body weight, adipose tissue and muscle weight were measured at the end of animal experiment (7th week).

Table 2. Effect of benfotiamine on blood glucose levels in OLETF rats

\begin{tabular}{ccccc}
\hline \hline Blood glucose level & LETO & OLETF & OLETF B100 & OLETF B200* $^{*}$ \\
\hline Initial level $(\mathrm{mg} / \mathrm{dL})^{\S}$ & $112 \pm 7.2$ & $135 \pm 12.3$ & $137 \pm 15.7$ & $142 \pm 11.4$ \\
Final level $(\mathrm{mg} / \mathrm{dL})^{\S}$ & $124 \pm 8.2$ & $182 \pm 12.4^{*}$ & $154 \pm 9.1^{\#}$ & $158 \pm 10.2^{\#}$ \\
\hline
\end{tabular}

Data are presented as the mean \pm SE. ${ }^{*} p<0.01 v s$. the LETO rats; ${ }^{*} p<0.05 v s$. the OLETF control. OLETF treated with $100 \mathrm{mg} / \mathrm{kg}$ benfotiamine. "OLETF treated with $200 \mathrm{mg} / \mathrm{kg}$ benfotiamine. ${ }^{8}$ Initial blood glucose levels were measured at Day 0 , and the final levels were measured at the end of animal experiment (7th week).

A

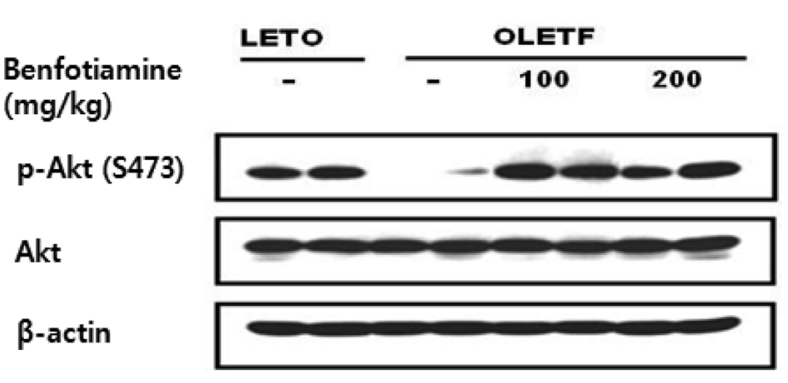

B

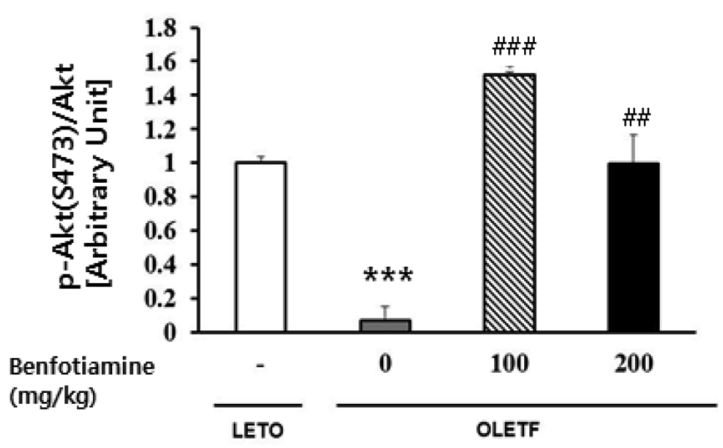

Fig. 1. Effects of benfotiamine on Akt phosphorylation (S473) in rat liver. (A) Akt phosphorylation (S473) was detected by immunoblotting. Representative results from two out of seven rats in each group are shown. (B) The ratio of p-Akt (S473) to Akt band was measured by densitometry. Values are expressed as the mean \pm SE. ${ }^{* * *} p<0.001 v s$. LETO rats, ${ }^{\# \#} p<0.01$ and $^{\# \# \#} p$ $<0.001 v s$. the OLETF control.
A

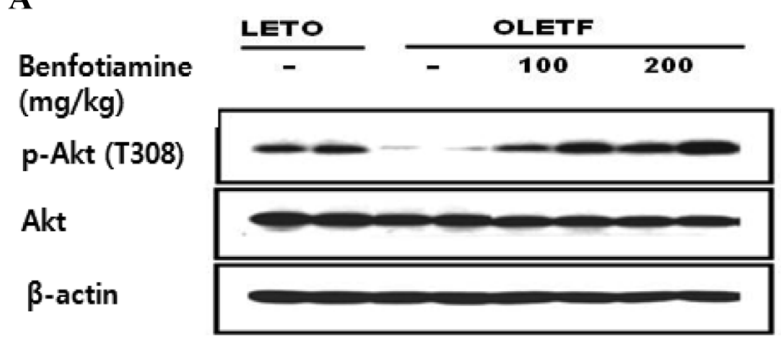

B

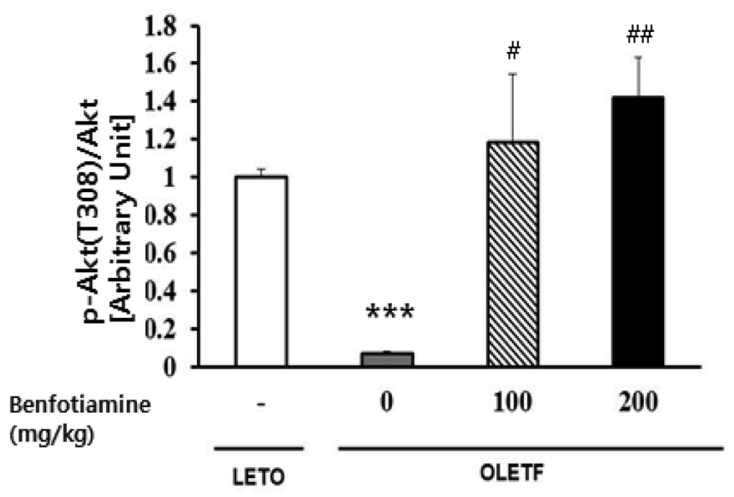

Fig. 2. Effects of benfotiamine on Akt phosphorylation (T308) in rat liver. (A) Akt phosphorylation (T308) was detected by immunoblotting. Representative results from two out of seven rats in each group are shown. (B) The ratio of p-Akt (T308) to Akt band was measured by densitometry. Values are expressed as the mean \pm SE. ${ }^{* * *} p<0.001 v s$. LETO rats, ${ }^{\sharp} p<0.05$ and ${ }^{\# \#} p$ $<0.01$ vs. the OLETF control. 
A

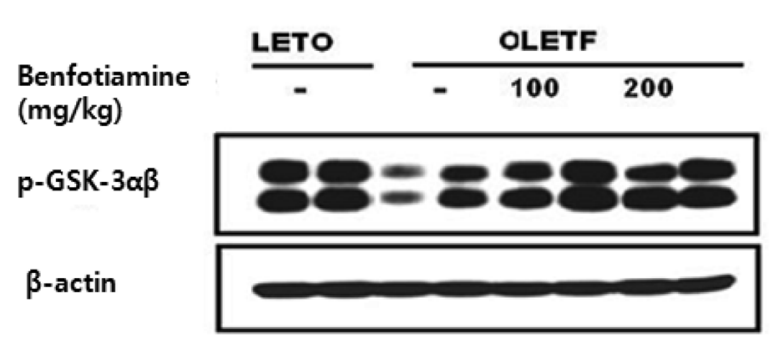

B

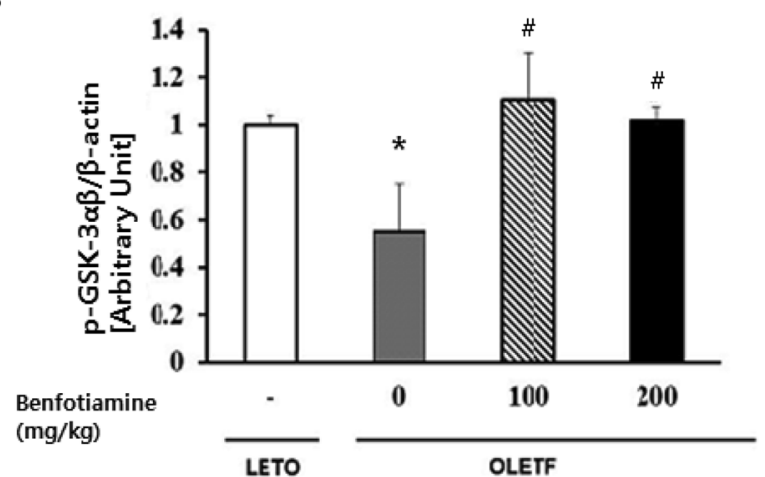

Fig. 3. Effects of benfotiamine on GSK-3 $\alpha / \beta$ phosphorylation $(\mathrm{S} 21 / \mathrm{S} 9)$ in rat liver. (A) GSK-3 $\alpha / \beta$ phosphorylation (S21/S9) was detected by immunoblotting. Representative results from two out of seven rats in each group are shown. (B) The ratio of p-GSK- $3 \alpha / \beta$ to $\beta$-actin band was measured by densitometry. Values represent the mean \pm SE. ${ }^{*} p<0.05$ vs. LETO rats, ${ }^{\sharp} p<$ 0.05 vs. the OLETF control.

by slowing down adipose tissue accumulation.

\section{Blood glucose levels}

The effect of benfotiamine on blood glucose levels was assessed before and after treatment with benfotiamine (day 0 and 7 week; Table 2). On day 0, blood glucose levels of the OLETF groups were slightly higher than those observed in the LETO group (Table 2). After 7 week, blood glucose levels of the OLETF control group were significantly greater $(p<0.01)$ than those in LETO group. These changes were significantly reduced $(p<0.05)$ by benfotiamine administration in the OLETF rats (Table 2). Our results indicate that benfotiamine reduces blood glucose levels in OLETF rats.

\section{Akt phosphorylation}

To observe the effects of benfotiamine on the insulin signaling pathway in the liver, Akt phosphorylation (S473/ T308) was monitored by Western blotting (Fig. 1 and 2). Akt phosphorylation levels of S473 in the OLETF control group were significantly lower $(p<0.001)$ than those observed in the LETO rats. After treatment with both concentrations of benfotiamine $(100 \mathrm{mg} / \mathrm{kg}$ and $200 \mathrm{mg} / \mathrm{kg})$, Akt phosphorylation of S473 increased significantly $(p<0.001$ and $p<0.01$,
A

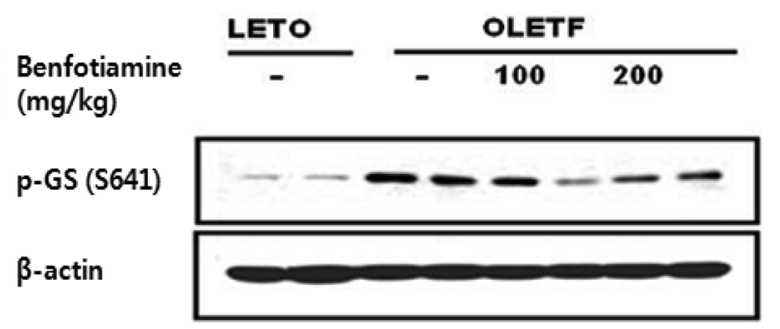

B

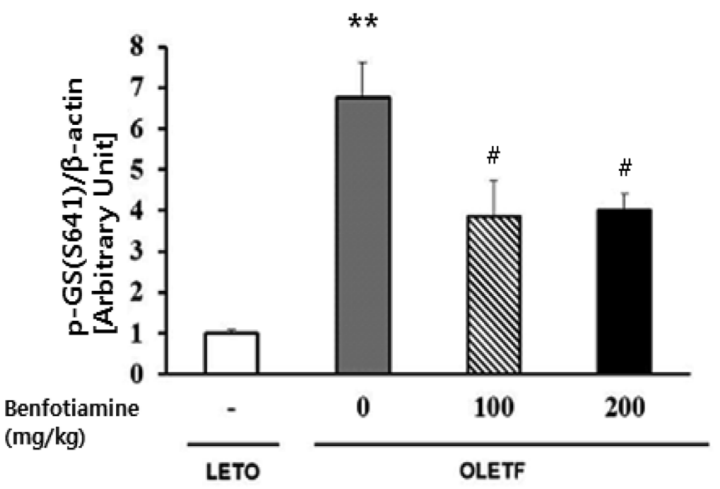

Fig. 4. Effects of benfotiamine on GS phosphorylation (S641) in rat liver. (A) Glycogen synthase (GS) phosphorylation (S641) was detected by immunoblotting. Representative results from two out of seven rats in each group are shown. (B) The ratio of p-GS (S641) to $\beta$-actin band was measured by densitometry. Values represent the mean \pm SE. ${ }^{* *} p<0.01$ vs. LETO rats, ${ }^{*} p<$ 0.05 vs. the OLETF control.

respectively) compared to OLETF control group so that they were even higher levels than those observed in the LETO group (Fig. 1). Similarly, Akt phosphorylation levels of T308 in the OLETF control group were significantly lower $(p<$ 0.001 ) than those observed in the LETO rats (Fig. 2). After treatment with benfotiamine $(100 \mathrm{mg} / \mathrm{kg}$ and $200 \mathrm{mg} / \mathrm{kg})$, Akt phosphorylation of T308 increased significantly $(p<$ 0.05 and $p<0.01$, respectively) compared to OLETF control group so that they were almost identical to the levels observed in the LETO group (Fig. 2). The results indicate that benfotiamine restores the phosphorylation of Akt to the normal levels in the liver of OLETF type 2 diabetic rats.

\section{GSK-3 $\alpha / \beta$ and GS phosphorylations}

The study also determined the effects of benfotiamine on the phosphorylation of downstream effectors in the insulin signaling pathway: GSK-3 $\alpha / \beta$ (S21/S9; Fig. 3) and GS (S641; Fig. 4). Even though the phosphorylation levels of GSK-3 $\alpha /$ $\beta$ in the OLETF control group were significantly lower $(p<$ $0.05)$ than those observed in the LETO rats, benfotiamine restored GSK- $3 \alpha / \beta$ phosphorylation in the OLETF rats $(p<$ 0.05 ) almost to the same levels observed in the LETO animals (Fig. 3). In contrast, the level of GS phosphorylation 
(S641) in the OLETF control group was significantly higher $(p<0.01)$ than that in the LETO group, the levels were significantly decreased $(p<0.05)$ in the OLETF treated with benfotiamine (Fig. 4). The results indicate that benfotiamine restores the phosphorylation of both GSK-3 $\alpha / \beta$ and GS to the normal levels in the liver of OLETF rats.

\section{Discussion}

The present study showed that benfotiamine reduced body weight gain, epididymal adipose tissue weight, and blood glucose levels in OLETF rats. The therapeutic effects of benfotiamine on type 2 diabetes mellitus in OLETF rats might result from the restoration of insulin sensitivity in the liver. Benfotiamine increased insulin sensitivity by increasing Akt phosphorylation and modulating the phosphorylation of downstream effector molecules. Phosphorylation of GSK-3 $\alpha$ / $\beta$ was increased by benfotiamine whereas GS phosphorylation was decreased.

Akt phosphorylation is important for glycogen synthesis in the liver [17]. A previous study observed that the phosphorylation level of Akt in adipose tissue from type 2 diabetic mice is decreased by $69 \%$ compared to control animals [24]. Growing evidence has suggested that benfotiamine ameliorates diabetic dysfunction via mechanisms involving the activation of Akt [10]. Indeed, benfotiamine was found to restore Akt phosphorylation and transketolase activity to normal levels in a mouse model of ischemic diabetic limbs [10]. The results of the present study suggest that benfotiamine can potentially attenuate type 2 diabetes mellitus in OLETF rats by restoring insulin sensitivity through the upregulation of Akt phosphorylation.

Once activated, Akt enters the cytoplasm where phosphorylates and inactivates GSK-3 [4, 5]. A major substrate of GSK-3 is GS, an enzyme that catalyzes the final step of glycogen synthesis $[4,5]$. GS phosphorylation by GSK-3 inhibits glycogen synthesis; therefore, the inactivation of GSK-3 by Akt promotes the storage of glucose as glycogen $[5,17]$. In contrast, the phosphorylation of Akt is decreased with insulin resistance and GSK-3 is activated $[6,16]$. Activated GSK-3 phosphorylates GS that cannot convert glucose to glycogen $[5,6]$. The results of the present study suggest that benfotiamine can attenuate type 2 diabetes mellitus in OLETF rats by restoring gluconeogenesis through the upregulation of GSK-3 $\alpha / \beta$ phosphorylation and the downregulation of GS phosphorylation.

Benfotiamine has been used to treat vascular complications associated with diabetes [25]. Although diabetic complications frequently occur with type 2 diabetes mellitus, the mechanism underlying the effect of benfotiamine on insulin resistance has not been fully elucidated. Benfotiamine, a thiamine derivative, can act as a coenzyme for thiamine-dependent transketolase which diverts excess fructose-6-phosphate and glycerhaldeyde-3-phosphate from glycolysis into the pentose-phosphate pathway, thus removing these potentially damaging metabolites from the cytosol $[2,12,22,25]$. Oxidative stress is therefore reduced by the transfer of upstream metabolites into the non-oxidative pentose-phosphate pathway. Since oxidative stress is an important factor of insulin resistance, benfotiamine might restore insulin sensitivity $[12$, 25]. Diabetes may be defined as a thiamine-deficient state because reduced thiamine/translocase activity has been described in diabetic patients [22]. Thiamine and/or its derivative, benfotiamine, have been found to counteract the damaging effects of hyperglycemia on vascular cells in vitro [12, 25].

Overall, these results suggest that benfotiamine can potentially attenuate type 2 diabetes mellitus in OLETF rats by restoring insulin sensitivity through the upregulation of Akt phosphorylation and the activation of two downstream signaling molecules, GSK-3 $\alpha / \beta$ and GS. Future studies should be conducted to investigate the mechanisms governing the effects of benfotimine on insulin resistance in other organs (e.g., skeletal muscle) and/or other insulin signaling factors (e.g., insulin receptor) in OLETF rats.

\section{Acknowledgments}

This research was supported by the 2013 scientific promotion program funded by Jeju National University, Korea.

\section{References}

1. Alberti KGMM, Zimmet P, Shaw J. The metabolic syndrome-a new worldwide definition. Lancet 2005, 366, 1059-1062.

2. Bitsch R, Wolf M, Möller J, Heuzeroth L, Grüneklee D. Bioavailability assessment of the lipophilic benfotiamine as compared to a water-soluble thiamine derivative. Ann Nutr Metab 1991, 35, 292-296.

3. Bradford MM. A rapid and sensitive method for the quantitation of microgram quantities of protein utilizing the principle of protein-dye binding. Anal Biochem 1976, 72, 248-254.

4. Brownlee M. Biochemistry and molecular cell biology of diabetic complications. Nature 2001, 414, 813-820.

5. Ciaraldi TP, Nikoulina SE, Bandukwala RA, Carter L, Henry RR. Role of glycogen synthase kinase- $3 \alpha$ in insulin action in cultured human skeletal muscle cells. Endocrinology 2007, 148, 4393-4399.

6. Cline GW, Johnson K, Regittnig W, Perret P, Tozzo E, Xiao L, Damico C, Shulman GI. Effects of a novel glycogen synthase kinase-3 inhibitor on insulin-stimulated glucose metabolism in Zucker diabetic fatty (fa/fa) rats. Diabetes 2002, 51, 2903-2910.

7. Diabetes Control and Complications Trial Research Group. The effect of intensive treatment of diabetes on the development and progression of long-term complication in insulin-dependent diabetes mellitus. N Engl J Med 1993, 329, 977-986.

8. Du XL, Edelstein D, Rossetti L, Fantus IG, Goldberg H, Ziyadeh F, Wu J, Brownlee M. Hyperglycemia-induced mitochondrial superoxide overproduction activates the hexosamine pathway and induces plasminogen activator 
inhibitor-1 expression by increasing Sp1 glycosylation. Proc Natl Acad Sci U S A 2000, 97, 12222-12226.

9. Frustaci A, Kajstura J, Chimenti C, Jakoniuk I, Leri A, Maseri A, Nadal-Ginard B, Anversa P. Myocardial cell death in human diabetes. Circ Res 2000, 87, 1123-1132.

10. Gadau S, Emanueli C, Van Linthout S, Graiani G, Todaro M, Meloni M, Campesi I, Invernici G, Spillmann F, Ward K, Madeddu P. Benfotiamine accelerates the healing of ischemic diabetic limbs in mice through protein kinase $\mathrm{B} /$ Akt-mediated potentiation of angiogenesis and inhibition of apoptosis. Diabetologia 2006, 49, 405-420.

11. Gardner CD, Eguchi S, Reynolds CM, Eguchi K, Frank GD, Motley ED. Hydrogen peroxide inhibits insulin signaling in vascular smooth muscle cells. Exp Biol Med 2003, 228, 836-842.

12. Hammes HP, Du X, Edelstein D, Taguchi T, Matsumura T, Ju Q, Lin J, Bierhaus A, Nawroth P, Hannak D, Neumaier M, Bergfeld R, Giardino I, Brownlee $M$. Benfotiamine blocks three major pathways of hyperglycemic damage and prevents experimental diabetic retinopathy. Nat Med 2003, 9, 294 -299.

13. Hays NP, Galassetti PR, Coker RH. Prevention and treatment of type 2 diabetes: current role of lifestyle, natural product, and pharmacological interventions. Pharmacol Ther 2008, 118, 181-191.

14. Hoehn KL, Salmon AB, Hohnen-Behrens C, Turner N, Hoy AJ, Maghzal GJ, Stocker R, Van Remmen H, Kraegen EW, Cooney GJ, Richardson AR, James JE. Insulin resistance is a cellular antioxidant defense mechanism. Proc Natl Acad Sci U S A 2009, 106, 17787-17792.

15. Kawano K, Hirashima T, Mori S, Saitoh Y, Kurosumi M, Natori T. Spontaneous long-term hyperglycemic rat with diabetic complications. Otsuka Long-Evans Tokushima Fatty (OLETF) strain. Diabetes 1992, 41, 1422-1428.

16. Leng Y, Karlsson HKR, Zierath JR. Insulin signaling defects in type 2 diabetes. Rev Endocr Metab Disord 2004, 5, 111-117.

17. MacAulay K, Doble BW, Patel S, Hansotia T, Sinclair EM, Drucker DJ, Nagy A, Woodgett JR. Glycogen synthase kinase $3 \alpha$-specific regulation of murine hepatic glycogen metabolism. Cell Metab 2007, 6, 329-337.

18. Nishikawa T, Edelstein D, Du XL, Yamagishi S, Matsumura T, Kaneda Y, Yorek MA, Beebe D, Oates PJ, Hammes HP, Giardino I, Brownlee M. Normalizing mitochondrial superoxide production blocks three pathways of hyperglycaemic damage. Nature 2000, 404, 787-790.

19. Nourooz-Zadeh J, Tajaddini-Surmadi J, McCarthy S, Betteridge DJ, Wolff SP. Elevated levels of authentic plasma hydroperoxides in NIDDM. Diabetes 1995, 44, 1054-1058.

20. Paolisso G, Giugliano D. Oxidative stress and insulin action: is there a relationship? Diabetologia 1996, 39, 357-363.

21. Park JL, Lee YS, Kim BH, Kang YH, Kim IJ, Kim YK, Son SM. Oxidative stress causes vascular insulin resistance in OLETF rat through increased IRS-1 degradation. J Korean Diabetes Assoc 2007, 31, 22-32.

22. Schenk G, Duggleby RG, Nixon PF. Properties and functions of the thiamine diphosphate dependent enzyme transketolase. Int J Biochem Cell Biol 1998, 30, 1297-1318.

23. Schmidt AM, Hori O, Brett J, Yan SD, Wautier JL, Stern D. Cellular receptors for advanced glycation end products. Implications for induction of oxidant stress and cellular dysfunction in the pathogenesis of vascular lesions. Arterioscler Thromb 1994, 14, 1521-1528.

24. Shao J, Yamashita H, Qiao L, Friedman JE. Decreased Akt kinase activity and insulin resistance in C57BL/KsJLeprdb/db mice. J Endocrinol 2000, 167, 107-115.

25. Stirban A, Negrean M, Stratmann B, Gawlowski T, Horstmann T, Götting C, Kleesiek K, Mueller-Roesel M, Koschinsky T, Uribarri H, Vlassara H, TSchoepe D. Benfotiamine prevents macro- and microvascular endothelial dysfunction and oxidative stress following a meal rich in advanced glycation end products in individuals with type 2 diabetes. Diabetes Care 2006, 29, 2064-2071.

26. Taniyama Y, Hitomi H, Shah A, Alexander RW, Griendling KK. Mechanisms of reactive oxygen species-dependent downregulation of insulin receptor substrate-1 by angiotensin II. Arterioscler Thromb Vasc Biol 2005, 25, 1142-1147.

27. Thorburn AW, Gumbiner B, Bulacan F, Wallace $P$, Henry RR. Intracellular Glucose oxidation and glycogen synthase activity are reduced in non-insulin-dependent (type II) diabetes independent of impaired glucose uptake. J Clin Invest 1990, 85, 522-529.

28. UK Prospective Diabetes Study (UKPDS) Group. Intensive blood-glucose control with sulphonylureas or insulin compared with conventional treatment and risk of complications in patients with type 2 diabetes (UKPDS 33). Lancet 1998, 352, 837-853. 